\title{
Prognostic models in papillary renal cell carcinoma
}

\author{
Kyrollis Attalla ${ }^{1}$, Martin H. Voss ${ }^{2}$, A. Ari Hakimi ${ }^{1}$ \\ ${ }^{1}$ Urology Service, Department of Surgery, ${ }^{2}$ Department of Medicine, Memorial Sloan Kettering Cancer Center, New York, NY, USA \\ Correspondence to: A Ari Hakimi, MD. 353 East 68th Street, 5th floor, New York, NY 10065, USA. Email: hakimia@mskcc.org. \\ Comment on: Klatte T, Gallagher KM, Afferi L, et al. The VENUSS prognostic model to predict disease recurrence following surgery for non- \\ metastatic papillary renal cell carcinoma: development and evaluation using the ASSURE prospective clinical trial cohort. BMC Med 2019;17:182.
}

Submitted May 06, 2020. Accepted for publication May 22, 2020.

doi: $10.21037 /$ atm-20-3750

View this article at: http://dx.doi.org/10.21037/atm-20-3750

Advances in the management of renal cell carcinoma (RCC) have yielded a better understanding of the distinct clinical factors related to both host and disease associated with prognosis and response to therapy, and the development of risk stratification tools has been accelerated by an increased understanding of tumor biology. The predominant histologic subtype of RCC, clear cell RCC (ccRCC) accounts for approximately $80 \%$ of renal neoplasms. Expectedly, the most widely employed risk stratification tools are drawn from large datasets that predominantly included ccRCC patients, and sensibly, risk stratification tools are most reliably used in patients with this variant (1-5). The notion that different histologic subtypes harbor varying biologic capacities has long been appreciated and has different implications in the early-stage and advanced setting. Indeed, large cohort studies of patients with nonmetastatic papillary RCC (PRCC) demonstrate favorable outcomes compared to ccRCC patients, while PRCC patients fared worse than their ccRCC counterparts in the metastatic setting $(6,7)$.

Given the relative rarity and varying degrees of malignant behavior among non-clear cell subtypes, the development of subtype-specific risk stratification tools remains understudied. In an effort to refine the prognostication of patients with PRCC-the second commonest RCC subtype-Klatte et al. developed VENUSS, a prognostic model for predicting disease recurrence postoperatively in patients with non-metastatic PRCC (8). The authors highlight that in contemporary practice, management and surveillance strategies, despite differences in disease biology and clinical behavior, are largely similar for patients with PRCC and ccRCC, owing to their occurrence in the same organ and the paucity of data which exists on the efficacy of available treatment options for PRCC. Again, PRCC differs from ccRCC on a molecular, clinical, and morphologic level, rendering the currently available prognostic models to be of relatively limited clinical utility in PRCC and highlighting this to be an area of need.

In a first step, Klatte et al. retrospectively examined a discovery cohort including 556 patients across five institutions who had undergone resection of non-metastatic (any T, any $\mathrm{N}$ stage) PRCC. A prognostic scoring system aimed at predicting disease recurrence was developed by integrating five factors significantly associated with risk of recurrence on multivariate analysis, which namely included venous tumour thrombus, nuclear grade, size, $\mathrm{T}$ and $\mathrm{N}$ stage. The VENUSS score was subsequently developed from coefficients of the model, ranging from 0 , the lowest score, to 11, the highest score. According to the VENUSS score, three risk groups were created, corresponding to a 5 -year cumulative incidence of recurrence of $2.9 \%$ in low-risk patients ( $0-2$ points), $15.4 \%$ in intermediaterisk patients (3-5 points) and $54.5 \%$ in high-risk patients (6 points or greater). Among low-risk patients, 91.7\% experienced oligometastatic disease recurrence, in contrast to $16.7 \%$ and $40 \%$ in intermediate- and high-risk patients, respectively. Higher c-indices were observed with the VENUSS score and group at every time point compared to various previously established models including the University of California Integrated Staging System (UISS), Tumor, Node, Metastasis (TNM), and the 2018 Leibovich groups $(9,10)$. Compared to the standard models, the VENUSS score and VENUSS group displayed a moderate net benefit in a decision curve analysis in threshold probabilities between $10-40 \%$.

Findings were then applied in a validation cohort 
consisting of the 150 PRCC patients recruited from all three treatment arms of the placebo-controlled ASSURE trial (Adjuvant Sunitinib or Sorafenib for Unfavorable Renalcell carcinoma, ECOG-ACRIN E2805, NCT00326898). In this cohort the authors computed recurrence risk based on VENUSS score and group and also applied the TNM, UISS, and 2018 Leibovich groups for comparison. Again, the VENUSS score and VENUSS group demonstrated discrimination superior to the comparison groups at every investigated time point.

The authors report interesting findings in the discovery cohort. Sixty patients had recurrent disease at a median of 12.5 months (IQR, 7-26.5 months) with a median postoperative follow-up time of 53 months. The cumulative incidence of disease recurrence was $5.9 \%$ at 1 year, $9 \%$ at 2 years, and $11.7 \%$ at 5 years. The first site of recurrent disease was the chest in 30 patients (50\%) and the abdomen in 49 patients $(81.7 \%)$, and 20 patients (33.3\%) developed their first relapse in both the chest and the abdomen. Interestingly, a subgroup analysis in 2019 by Narayan et al. examining patterns of relapse in 403 high-risk non-ccRCC patients from the ASSURE trial demonstrated similar patterns of relapse (11). Abdominal sites of recurrence were significantly more likely to occur among patients with nonccRCC (5-year recurrence rate $26.4 \%$ vs. $18.2 \%, \mathrm{P}=0.0008$ ) and relapse in the chest was less likely to occur in patients with non-ccRCC (5-year recurrence rate $13.7 \%$ vs. $20.9 \%$, $\mathrm{P}=0.0005$ ) compared to patients with ccRCC.

It is helpful to look closer at the established risk stratification tools (including the ones used by Klatte et al. for comparison) and appreciate their limited applicability to patients with PRCC. The TNM staging system, for instance, considers tumor size indirectly through $\mathrm{T}$ stage and is not specific to PRCC and disregards subtype-specific biologic capacities. The development of UISS included patients with all RCC subtypes, the predominance of which was ccRCC. Likewise, the Grade, Age, Nodes and Tumor (GRANT) score was developed for both clear cell and nonclear cell RCC from the ASSURE trial cohort (12). A 2010 nomogram predicting cancer-specific survival in PRCC patients was developed by the same primary author, Klatte et al., however patients with and without distant metastases were included (13). Most recently in 2018, Leibovich et al. performed a cohort review of 3,633 patients from the Mayo Clinic Nephrectomy registry, 607 (17\%) of whom had PRCC, and developed a model for PRCC in which three prognostic groups were created for progression-free and cancer-specific survival (9). The model is limited in that it lacks external validation, calibration, and an assessment of clinical net benefits.

The VENUSS prognostic model differs from many of the aforementioned models in that it includes a relatively large cohort of PRCC patients and specifically examines risk of recurrence post-surgery in non-metastatic patients. Further, superior discrimination, calibration, and clinical net benefit from the VENUSS model was seen compared to the UISS, TNM and Leibovich prognostic groups. Klatte and colleagues are commended for a well-performed study analyzing one of the largest cohorts of non-metastatic PRCC and addressing an unmet need in the current body of literature. The authors recognize several important limitations; the validation cohort displayed poorer discrimination and calibration than in the discovery cohort, likely secondary to a relatively non-comparable discovery cohort. The discovery cohort was comprised of two-thirds patients with stage I disease and included consecutive patients, compared to only $10 \%$ of patients in ASSURE (validation cohort) who were recruited from a group of prescreened patients with a higher risk of disease recurrence. Almost two-thirds of the validation cohort received adjuvant tyrosine kinase inhibitor therapy with sunitinib or sorafenib, and although ASSURE neither achieved its primary endpoint of reducing disease free survival across all patients nor did subgroup analyses suggest it did in non-ccRCC, one cannot safely assume that it had no impact on disease recurrence. These discrepancies may have allowed for the observed differences in c-indices and calibration, which the authors cite is dependent on variation of predictors.

Follow-up was not standardized across all centers in the discovery cohort, nor was the decision to perform lymph node dissection at time of surgery which may potentially result in missed recurrences or the misclassification of nodal stage, as a percentage of patients are likely to harbor microscopic, radiographically benign lymph nodes (the true prevalence of which remains unknown, as a cohort of such patients undergoing templated lymph node dissection has not been studied). Perhaps most importantly, the authors cite the lack of central pathology review across the five institutions from which the discovery cohort was derived. The assertion that lack of central review exemplifies "real world" scenarios wherein central pathological review is rarely performed lends opportunities for considerable underreporting of pathologic features. While this may be true, developing sound prognostic models should likely be performed under strict standardization and stringent criteria for pathologic classifications (i.e., under central review), as this would 
potentially avoid missing key candidate pathologic variables. Sarcomatoid features were not demonstrated as a significant prognostic variable on multivariable analysis, emphasizing the concern predicated around the lack of centralized review. RCC with sarcomatoid differentiation occurs in $2-3 \%$ of PRCC with a high propensity for primary metastasis, with approximately $75 \%$ of patients with sarcomatoid differentiation presenting with metastatic disease (14-16). Further, the presence of even small tumoral components of sarcomatoid differentiation was demonstrated to be an independent predictor of poor survival compared to patients with RCC lacking sarcomatoid features $(14,17)$. Tumor necrosis was also not found to be a significant prognostic variable on multivariable analysis in the VENUSS model, and similarly, studies suggest presence of tumor necrosis to confer worse recurrence-free, progression-free, cancerspecific, and overall-survival (18). Additionally, it is unclear if quantifying the extent of tumor necrosis or sarcomatoid differentiation associates with outcome.

PRCC is routinely divided into type 1 , which characteristically has low-grade nuclei, and type 2, which has higher-grade nuclei, among other morphologic differences. Many institutions and pathologists, however, have strayed away from type 1 or type 2 designations, as the histologicallydefined type 2 PRCC encompasses a heterogenous spectrum of tumor morphologies. Likewise, various non-ccRCC subtypes mimic type 2 PRCC by displaying prominent papillary architecture. These subtypes include hereditary leiomyomatosis and RCC (HLRCC), MiT translocation RCC, collecting duct carcinoma, and more recently defined entities including acquired cystic disease-associated RCC, resulting in inconsistencies in reporting among pathologists (19). Recent genetic characterizations of these tumors further demonstrates type 2 PRCC to be a diverse collection of tumors which can be parsed into at least three subtypes (20). This is in contrast to sporadic type 1 PRCC, where nearly universal gains or trisomy of chromosomes 7 and 17 are observed. In the development of VENUSS, data on PRCC type 1 versus type 2 was collected on 493 of 556 patients $(89 \%)$ in the multi-institutional discovery cohort, and papillary type was not found to be a significant prognosticator on multivariable analysis. This is unexpected and may relate to heterogeneity in pathology reporting of PRCC among the five institutions from which data was collected, again underscoring the potential issues surrounding lack of central pathology review.

Prognostic models such as VENUSS are designed to be critical components of clinical trial design, risk-directed adjuvant therapy, postoperative surveillance, and patient counseling. This study has addressed a true area of need and hence is an important and welcome addition to the growing body of research addressing risk-stratification in non-ccRCC. The authors must be applauded for meeting the challenge of constructing the first dedicated prognostic model in nonmetastatic PRCC, and its use in this setting is encouraged. Looking onwards, VENUSS, as with many preceding models, critically serves as a substrate for further iterative refinements of discriminatory power through the reassessment and likely integration of pathologic, clinical, and genomic factors as further insights are gained into underlying tumor biology.

\section{Acknowledgments}

Funding: None.

\section{Footnote}

Provenance and Peer Review: This article was commissioned and reviewed by the Section Editor Dr. Xiao Li (Department of Urology, Jiangsu Cancer Hospital, Jiangsu Institute of Cancer Research, Nanjing Medical University Affiliated Cancer Hospital, Nanjing, China).

Conflicts of Interest: All authors have completed the ICMJE uniform disclosure form (available at http://dx.doi. org/10.21037/atm-20-3750). MHV reports personal fees from Novartis, personal fees from Alexion Pharmaceuticals, personal fees from Bayer, personal fees from Calithera Biosciences, personal fees from Corvus Pharmaceuticals, personal fees from Exelixis, personal fees and non-financial support from Eisai, personal fees from GlaxoSmithKline, personal fees from Natera, personal fees and non-financial support from Novartis, grants and personal fees from Pfizer, non-financial support from Takeda, grants from BristolMyers Squibb, grants from Genentech/Roche, outside the submitted work. The other authors have no conflicts of interest to declare.

Ethical Statement: The authors are accountable for all aspects of the work in ensuring that questions related to the accuracy or integrity of any part of the work are appropriately investigated and resolved.

Open Access Statement: This is an Open Access article distributed in accordance with the Creative Commons Attribution-NonCommercial-NoDerivs 4.0 International 


\section{Page 4 of 4}

License (CC BY-NC-ND 4.0), which permits the noncommercial replication and distribution of the article with the strict proviso that no changes or edits are made and the original work is properly cited (including links to both the formal publication through the relevant DOI and the license). See: https://creativecommons.org/licenses/by-nc-nd/4.0/.

\section{References}

1. Motzer RJ, Mazumdar M, Bacik J, et al. Survival and prognostic stratification of 670 patients with advanced renal cell carcinoma. J Clin Oncol 1999;17:2530-40.

2. Heng DY, Xie W, Regan MM, et al. Prognostic factors for overall survival in patients with metastatic renal cell carcinoma treated with vascular endothelial growth factortargeted agents: results from a large, multicenter study. J Clin Oncol 2009;27:5794-9.

3. Mekhail TM, Abou-Jawde RM, Boumerhi G, et al. Validation and extension of the Memorial Sloan-Kettering prognostic factors model for survival in patients with previously untreated metastatic renal cell carcinoma. J Clin Oncol 2005;23:832-41.

4. Heng DY, Xie W, Regan MM, et al. External validation and comparison with other models of the International Metastatic Renal-Cell Carcinoma Database Consortium prognostic model: a population-based study. Lancet Oncol 2013;14:141-8.

5. Negrier S, Escudier B, Gomez F, et al. Prognostic factors of survival and rapid progression in 782 patients with metastatic renal carcinomas treated by cytokines: a report from the Groupe Francais d'Immunotherapie. Ann Oncol 2002;13:1460-8.

6. Wagener N, Edelmann D, Benner A, et al. Outcome of papillary versus clear cell renal cell carcinoma varies significantly in non-metastatic disease. PLoS One 2017;12:e0184173.

7. Connor Wells J, Donskov F, Fraccon AP, et al. Characterizing the outcomes of metastatic papillary renal cell carcinoma. Cancer Med 2017;6:902-9.

8. Klatte T, Gallagher KM, Afferi L, et al. The VENUSS prognostic model to predict disease recurrence following surgery for non-metastatic papillary renal cell carcinoma: development and evaluation using the ASSURE prospective clinical trial cohort. BMC Med 2019;17:182.

9. Leibovich BC, Lohse CM, Cheville JC, et al. Predicting Oncologic Outcomes in Renal Cell Carcinoma After Surgery. Eur Urol 2018;73:772-80.

10. Haas NB, Manola J, Uzzo RG, et al. Adjuvant sunitinib or

\section{Attalla et al. VENUSS: a proposed PRCC prognostic model}

sorafenib for high-risk, non-metastatic renal-cell carcinoma (ECOG-ACRIN E2805): a double-blind, placebo-controlled, randomised, phase 3 trial. Lancet 2016;387:2008-16.

11. Narayan V, Puligandla M, Haas NB, et al. Patterns of Relapse and Implications for Post-Nephrectomy Surveillance in Patients with High Risk Nonclear Cell Renal Cell Carcinoma: Subgroup Analysis of the Phase 3 ECOG-ACRIN E2805 Trial. J Urol 2019;201:62-8.

12. Buti S, Puligandla M, Bersanelli M, et al. Validation of a new prognostic model to easily predict outcome in renal cell carcinoma: the GRANT score applied to the ASSURE trial population. Ann Oncol 2017;28:2747-53.

13. Klatte T, Remzi M, Zigeuner RE, et al. Development and external validation of a nomogram predicting disease specific survival after nephrectomy for papillary renal cell carcinoma. J Urol 2010;184:53-8.

14. de Peralta-Venturina M, Moch H, Amin M, et al. Sarcomatoid differentiation in renal cell carcinoma: a study of 101 cases. Am J Surg Pathol 2001;25:275-84.

15. Mian BM, Bhadkamkar N, Slaton JW, et al. Prognostic factors and survival of patients with sarcomatoid renal cell carcinoma. J Urol 2002;167:65-70.

16. Merrill MM, Wood CG, Tannir NM, et al. Clinically nonmetastatic renal cell carcinoma with sarcomatoid dedifferentiation: Natural history and outcomes after surgical resection with curative intent. Urol Oncol 2015;33:166.e21-9.

17. Pichler R, Comperat E, Klatte T, et al. Renal Cell Carcinoma with Sarcomatoid Features: Finally New Therapeutic Hope? Cancers (Basel) 2019;11.

18. Zhang L, Zha Z, Qu W, et al. Tumor necrosis as a prognostic variable for the clinical outcome in patients with renal cell carcinoma: a systematic review and metaanalysis. BMC Cancer 2018;18:870.

19. Signoretti S, Flaifel A, Chen YB, et al. Renal Cell Carcinoma in the Era of Precision Medicine: From Molecular Pathology to Tissue-Based Biomarkers. J Clin Oncol 2018:JCO2018792259.

20. Cancer Genome Atlas Research N, Linehan WM, Spellman PT, et al. Comprehensive Molecular Characterization of Papillary Renal-Cell Carcinoma. N Engl J Med 2016;374:135-45.

Cite this article as: Attalla K, Voss MH, Hakimi AA Prognostic models in papillary renal cell carcinoma. Ann Transl Med 2020;8(21):1334. doi: 10.21037/atm-20-3750 